\title{
Predictors of mortality and cardiovascular outcomes in Emery-Dreifuss muscular dystrophy in a long-term follow-up
}

\author{
Michał Marchel' ', Agnieszka Madej-Pilarczyk², Roman Steckiewicz' , Przemysław Stolarz', Michał Peller', \\ Agata Tymińska' , Ewa Ostrowska' , Krzysztof Ozierański', Paweł Balsam¹, Marcin Grabowski', \\ Grzegorz Opolski ${ }^{1}$
}

${ }^{1} 1^{\text {st }}$ Chair and Department of Cardiology, Medical University of Warsaw, Warszawa, Poland

${ }^{2}$ Department of Medical Genetics, The Children's Memorial Health Institute, Warszawa, Poland

\author{
Correspondence to: \\ Michał Marchel, MD, PhD, \\ $1^{\text {st }}$ Chair and Department \\ of Cardiology, \\ Medical University of Warsaw, \\ Banacha 1A, 02-097 Warszawa, \\ Poland, \\ phone: +48 225992958 , \\ e-mail: \\ michal.marchel@wum.edu.pl \\ Copyright by the Author(s), 2021 \\ Kardiol Pol. 2021; \\ 79 (12): 1335-1342; \\ DOI: 10.33963/KP.a2021.0159 \\ Received: \\ August 4, 2021 \\ Revision accepted: \\ November 14, 2021 \\ Published online: \\ November 16, 2021
}

\begin{abstract}
Background: Emery-Dreifuss muscular dystrophy (EDMD) is an extremely rare muscular dystrophy due to either emerinopathy $(E M D)$ or laminopathy $(L M N A)$. The main risk for patients is that of cardiovascular complications.
\end{abstract}

Aims: This study aimed to identify predictors of adverse clinical events in patients with EDMD in a long-term follow-up observation.

Methods: A total of 45 patients with confirmed EMD or LMNA mutation were included in the study. The relationships between clinical parameters, the overall survival rate, and risk factors for disease progression were assessed. The primary endpoint was defined as death, while the secondary endpoint comprised death, resuscitated cardiac arrest (RCA), heart transplant (HTX), stroke, end-stage heart failure (ESHF), and hospitalization due to heart failure (HF).

Results: During a median length of follow-up observation of ten years (interquartile range, 5-15), ten patients (22\%) died, one suffered RCA, two had HTX, and six suffered ischemic strokes (13\%). Seven patients developed ESHF, and eight were hospitalized due to HF. The secondary endpoint occurred in 16 patients (36\%). LMNA mutation (hazard ratio [HR], $6.01 ; 95 \%$ confidence interval [CI], 1.61-22.4; $P=0.008$ ) and higher serum $N$-terminal fragment of B-type natriuretic peptide (NT-proBNP) concentration ( $\mathrm{HR}, 1.29 ; 95 \% \mathrm{Cl}, 1.06-1.56 \mathrm{per} 100 \mathrm{pg} / \mathrm{ml} ; P=0.01)$ increased the risk of death. Higher tricuspid annular plane systolic excursion (TAPSE) decreased the risk for the secondary endpoint (HR, $0.78 ; 95 \% \mathrm{Cl}, 0.68-0.90 \mathrm{~mm} ; P<0.001)$. NT-proBNP $>257 \mathrm{pg} / \mathrm{ml}$ and TAPSE $<21 \mathrm{~mm}$ may be assumed as the best cut-off values for the primary and secondary endpoints, respectively.

Conclusions: LMNA mutation and higher NT-proBNP concentration were associated with increased mortality in EDMD. Lower TAPSE was a predictor of a composite secondary endpoint in EDMD.

Key words: EMD, cardiomyopathy, heart failure, laminopathy, LMNA

Kardiol Pol 2021; 79, 12: 1335-1342

\section{INTRODUCTION}

Emery-Dreifuss muscular dystrophy (EDMD) is an extremely rare form of muscular dystrophy due to either emerinopathy (EDMD1; mutation in the EMD gene) or laminopathy (EDMD2; mutation in the LMNA gene) [1]. Both most commonly mutated proteins are components of the nuclear envelope. The peripheral muscle's involvement might be benign while the main risk for patients is that of cardiovascular complications [2]. Features of typical cardiomyopathy [3] and typical arrhythmias occurring during the disease [4] have been previously described. The current study aimed to identify predictors of adverse clinical events in a long-term follow-up observation. 
WHAT'S NEW?

Emery-Dreifuss muscular dystrophy (EDMD) is an extremely rare form of muscular dystrophy due to either emerinopathy (EMD) or laminopathy (LMNA). The main risk for patients is that of cardiovascular complications. Our findings show that patients with the presence of LMNA mutation and N-terminal fragment of B-type natriuretic peptide (NT-proBNP) concentration $>257 \mathrm{pg} / \mathrm{ml}$ were associated with increased mortality in the long-term follow-up observation, with a $29 \%$ increase in the risk of death for each $100 \mathrm{pg} / \mathrm{ml}$ of NT-proBNP increase. Tricuspid annular plane systolic excursion <21 mm was an additional predictor of future adverse events and increased cardiovascular risk in EDMD, indicating the need for clinical vigilance, even in the case of mildly diminished right ventricular function.

\section{METHODS}

This analysis is based on a prospective, observational, single-center study, conducted between 2000 and 2021. A total of 45 consecutive patients with muscular dystrophy and confirmed EMD or LMNA mutation referred to the $1^{\text {st }}$ Department of Cardiology, Medical University of Warsaw were included in the study. Clinical and echocardiographic parameters as well as cardiac biomarkers were assessed at baseline and then correlated with the clinical course of the disease. Clinical parameters included: heart failure symptoms, atrioventricular (AV) block (determined based on electrocardiographic criteria), atrial fibrillation (paroxysmal, persistent, and permanent), and atrial standstill [4]. The last one, characterized by the absence of electrical and mechanical activity of the atria, is one of the features of EDMD. At inclusion, the transthoracic echocardiography was performed in all subjects. All four heart chambers diameters and volumes, as well as the systolic function of both right and left ventricles, were measured according to the protocol previously described [3]. Venous blood samples were obtained at inclusion, and plasma levels of cardiac biomarkers (NT-proBNP, Roche Diagnostics GmbH, Mannheim, Germany; NT-proANP, Biomedica Medizinprodukte ${ }^{\oplus} \mathrm{GmbH}$, Wien, Austria) were determined. Once a year a control visit in our department or telephone contact was conducted to collect follow-up data. Patient data for the study were collected based on documentation from the primary care physician. At the on-site visit, additional clinical examination and echocardiography were performed. The primary endpoint was defined as all-cause death, while the secondary endpoint was a composite of all-cause death, resuscitated cardiac arrest (RCA), heart transplant (HTX), stroke, end-stage heart failure (ESHF), and hospitalization for heart failure (HF). All endpoints were assessed and confirmed based on the patient's medical records. Predictors of the primary and secondary endpoints were identified.

\section{Statistical analysis}

Distributions of continuous variables were checked using the Shapiro-Wilk test. All continuous variables were non-normally distributed and presented as medians and interquartile ranges (IQRs). For categorical variables, absolute and relative frequencies were presented. Differ- ences between groups for categorical and continuous variables were calculated using Fisher's exact test and the Mann-Whitney U test, respectively. Risk factors for particular endpoints were calculated using univariate and stepwise multivariable Cox regression analysis. Patient outcomes were presented with Kaplan-Meier curves. Receiver operating characteristic (ROC) curves were constructed to assess the predictive value of NT-proBNP and TAPSE for the primary and secondary endpoint, respectively. Cut-off points were calculated based on the Youden index. The value of $P<0.05$ was considered significant for all tests. All tests were two-tailed. Statistical analysis was performed using SAS ${ }^{\oplus}$ software, version 9.4 (Cary, NC, USA).

The study was approved by the Local Ethics Committee $(K B / 2 / 2005)$ of the Medical University of Warsaw and was performed in accordance with the 1976 Declaration of Helsinki and its later amendments. All patients signed informed consent forms before participating in the study.

\section{RESULTS}

The baseline characteristics of the studied population are presented in Tables 1 and 2. The median age at inclusion was 25 (IQR, 16-38) years. One-third of our cohort were patients with EDMD2. In the EDMD1 group, 20\% of patients were females (only 1 in 5 female carriers of EDMD1 presented cardiac involvement), while in the EDMD2 group $73 \%$. All patients with mild HF symptoms at baseline evaluation were EDMD2. There were no differences in terms of the number of sporadic and familial cases, atrial fibrillation, AV block, baseline treatment, and pacemaker implantation between the two main EDMD groups. A trend towards a higher percentage of $\beta$-blockers usage in the EDMD2 group is worth noting. The neurological status was also comparable between the two EDMD groups, as in each of them, there were both patients with minimal skeletal muscle involvement but also with generalized muscle atrophy and profound joint contractures, who require support in everyday life. Only one female patient with EDMD2 was wheelchair-bound. Due to the relatively young median age, no other cardiovascular risk factors were identified. During the median length of the ten-year (IQR, 5-15) follow-up observation, the primary endpoint occurred in ten patients (22\%). Components of the secondary endpoint occurred 
Table 1. Baseline characteristics of the studied population depending on the type of mutation

\begin{tabular}{|c|c|c|c|}
\hline & $\begin{array}{l}\text { EDMD1 (EMD) } \\
\quad(\mathrm{n}=\mathbf{3 0})\end{array}$ & $\begin{array}{l}\text { EDMD2 (LMNA) } \\
\quad(n=15)\end{array}$ & P-value \\
\hline Age, year, median (IQR) & $21.0(15.25-30.0)$ & $26.0(18.0-33.0)$ & 0.29 \\
\hline Female gender, n (\%) & $6(20)$ & $11(73.3)$ & $<0.001$ \\
\hline $\mathrm{BMI}, \mathrm{kg} / \mathrm{m}^{2}$, median (IQR) & $21.5(19.4-25.2)$ & $20.2(17.3-25.1)$ & 0.36 \\
\hline Sporadic/familial, n (\%) & $13(43.3) / 17(56.7)$ & $9(60) / 6(40)$ & 0.15 \\
\hline \multicolumn{4}{|l|}{ Muscular involvement } \\
\hline Mild, n (\%) & $13(43.3)$ & $8(53.3)$ & 0.66 \\
\hline Moderate, n (\%) & $14(46.7)$ & $5(33.3)$ & \\
\hline Severe, n (\%) & $3(10)$ & $2(13.3)$ & \\
\hline Heart failure, n (\%) & $0(0)$ & $3(20)$ & 0.03 \\
\hline Atrial fibrillation, n (\%) & $10(33.3)$ & $3(20)$ & 0.49 \\
\hline AV block, n (\%) & $20(66.7)$ & $9(60)$ & 0.75 \\
\hline LVEF, \%, median (IQR) & $52(48-58)$ & $54(48-58)$ & 0.94 \\
\hline NT-proBNP, pg/ml, median (IQR) & $70(44-102)$ & $109(54-347)$ & 0.13 \\
\hline ACE inhibitors, $n$ (\%) & $14(46.7)$ & $9(60)$ & 0.53 \\
\hline$\beta$-blockers, n (\%) & $6(20)$ & $6(40)$ & 0.17 \\
\hline Anticoagulants, n (\%) & $10(33.3)$ & $4(26.7)$ & 0.74 \\
\hline Pacemaker, n (\%) & $19(63.3)$ & $7(46.7)$ & 0.35 \\
\hline
\end{tabular}

Abbreviations: $A C E$, angiotensin-converting-enzyme; $A V$, atrioventricular; $B M I$, body mass index; $E M D$, mutation in EMD gene; IQR, interquartile range; $L V E F$, left ventricular ejection fraction; LMNA, mutation in LMNA gene; NT-proBNP, N-terminal prohormone B-type natriuretic peptide; NYHA, New York Heart Association class

Table 2. Baseline characteristics and the primary and secondary composite endpoints

\begin{tabular}{|c|c|c|c|c|c|c|c|}
\hline \multirow[t]{2}{*}{ Variable } & \multirow{2}{*}{$\begin{array}{c}\text { Total } \\
(n=45)\end{array}$} & \multicolumn{3}{|c|}{ Primary endpoint } & \multicolumn{3}{|c|}{ Secondary endpoint } \\
\hline & & $\begin{array}{c}\text { No } \\
(n=35)\end{array}$ & $\begin{array}{c}\text { Yes } \\
(n=10)\end{array}$ & $P$-value & $\begin{array}{c}\text { No } \\
(n=29)\end{array}$ & $\begin{array}{c}\text { Yes } \\
(n=16)\end{array}$ & $P$-value \\
\hline Age, years, median (IQR) & $25(16-38)$ & $25(16-38)$ & $26(18-40)$ & 0.90 & $25(16-38)$ & $27(21-39)$ & 0.50 \\
\hline Female gender, $\mathrm{n}(\%)$ & $38(17)$ & $34(12)$ & $50(5)$ & 0.50 & $34(10)$ & $44(7)$ & 0.70 \\
\hline LMNA mutation, $\mathrm{n}(\%)$ & $33(15)$ & $26(9)$ & $60(6)$ & 0.06 & $21(6)$ & $56(9)$ & 0.02 \\
\hline Non-miss-sense mutation, $\mathrm{n}(\%)$ & $4(2)$ & $6(2)$ & $0(0)$ & 1.00 & $0(0)$ & $13(2)$ & 0.10 \\
\hline NYHA (II-IV), n (\%) & $7(3)$ & $6(2)$ & $10(1)$ & 0.50 & $0(0)$ & $19(3)$ & 0.04 \\
\hline Sinus rhythm, $\mathrm{n}(\%)$ & $84(38)$ & $89(31)$ & $70(7)$ & 0.20 & $93(27)$ & $69(11)$ & 0.08 \\
\hline Atrial fibrillation, $\mathrm{n}(\%)$ & $29(13)$ & $31(11)$ & $20(2)$ & 0.70 & $28(8)$ & $31(5)$ & 1.00 \\
\hline AV block, n (\%) & $64(29)$ & $60(21)$ & $80(8)$ & 0.30 & $55(16)$ & $81(13)$ & 0.10 \\
\hline NT-proBNP, pg/ml, median (IQR) & $78(51-213)$ & $70(43-116)$ & $295(71-411)$ & 0.02 & $67(42-102)$ & $256(71-411)$ & 0.01 \\
\hline NT-proANP, pmol/l, median (IQR) & $1.02(0.82-1.73)$ & $0.958(0.821-1.59)$ & $1.63(0.931-2.07)$ & 0.40 & $0.937(0.817-1.30)$ & $1.65(0.865-2.60)$ & 0.10 \\
\hline LVEDdi, mm/m², median (IQR) & $30(27-32)$ & $29(26-32)$ & $32(30-33)$ & 0.05 & $28(26-31)$ & $32(30-33)$ & 0.02 \\
\hline LVESdi, $\mathrm{mm} / \mathrm{m}^{2}$, median (IQR) & $19(16-22)$ & $19(15-21)$ & $23(21-25)$ & 0.01 & $17(15-21)$ & $22(20-25)$ & 0.003 \\
\hline LADi, mm/m², median (IQR) & $21(19-23)$ & $21(19-24)$ & $21(19-23)$ & 1.00 & $21(19-23)$ & $22(20-25)$ & 0.30 \\
\hline LAVi, ml/m², median (IQR) & $34(26-39)$ & $33(26-40)$ & $36(27-37)$ & 0.80 & $32(25-38)$ & $37(33-43)$ & 0.08 \\
\hline LVEF, \%, median (IQR) & $53(48-59)$ & $53(49-59)$ & $52(40-57)$ & 0.50 & $54(49-60)$ & $51(40-57)$ & 0.20 \\
\hline LVSF, \%, median (IQR) & $35(27-44)$ & $35(31-45)$ & $25(22-36)$ & 0.02 & $37(32-45)$ & $26(22-36)$ & 0.02 \\
\hline $\mathrm{RVi}, \mathrm{mm} / \mathrm{m}^{2}$, median (IQR) & $14(12-16)$ & $13(12-16)$ & $15(12-16)$ & 0.60 & $13(11-16)$ & $14(12-16)$ & 0.40 \\
\hline $\mathrm{RAVi}, \mathrm{ml} / \mathrm{m}^{2}$, median (IQR) & $38(28-56)$ & $32(27-56)$ & $46(38-46)$ & 0.20 & $30(25-49)$ & $52(44-107)$ & 0.004 \\
\hline TAPSE, mm, median (IQR) & $22(19-25)$ & $24(21-26)$ & $18(16-21)$ & 0.004 & $24(22-26)$ & $18(16-21)$ & $<0.001$ \\
\hline TVPG, mm Hg, median (IQR) & $20(12-30)$ & $19(6-29)$ & $29(26-33)$ & 0.10 & $18(0-24)$ & $30(26-40)$ & 0.005 \\
\hline
\end{tabular}

Abbreviations: LADi, left atrial diameter indexed for body surface area; LAVi, left atrial volume indexed for body surface area; LVEDdi, left ventricular end-diastolic diameter indexed for body surface area; LVESdi, left ventricular end-systolic diameter indexed for body surface area; LVSF, left ventricular systolic function; NT-proANP, N-terminal fragment of A-type natriuretic peptide; RAVi, right atrial volume indexed for body surface area; RVi, right ventricular diameter indexed for body surface area; TAPSE, tricuspid annular plane systolic excursion; TVPG, tricuspid valve pressure gradient; other - see Table 1

as follows: $\mathrm{RCA}-\mathrm{n}=1(2 \%), \mathrm{HTX}-\mathrm{n}=2(4 \%)$, ischemic stroke occurred $-n=6(13 \%), E S H F-n=7(16 \%)$, hospitalization due to $\mathrm{HF}-\mathrm{n}=8(18 \%)$. A total of 34 secondary endpoints occurred in 16 patients (36\%) (Figure 1).

Kaplan-Meier curves for the primary and secondary endpoints for patients with both emerinopathy (EDMD1) and laminopathy (EDMD2) are presented in Figure 2.
Patients who died had higher N-terminal of the prohormone brain natriuretic peptide (NT-proBNP) serum concentrations, higher indexed end-systolic left ventricular dimension (LVESdi), lower left ventricular shortening fraction (LVSF), and worse right ventricular function estimated by tricuspid annular plane systolic excursion (TAPSE). Additionally, those who experienced the com- 


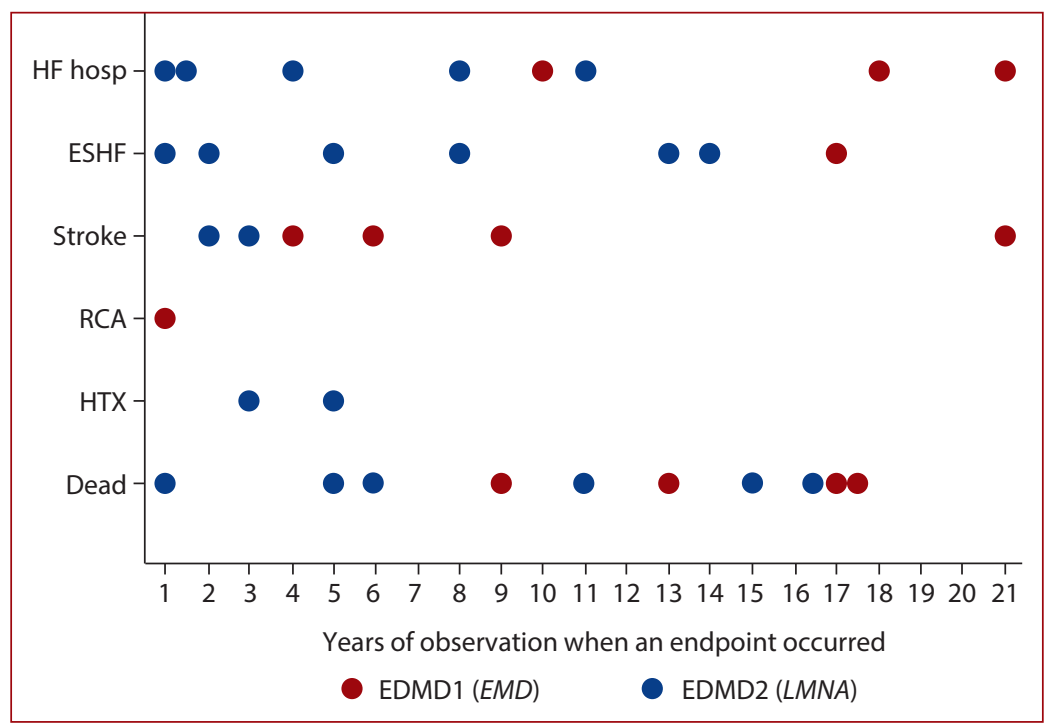

Figure 1. Timeline of all endpoint occurrences during the follow-up

Abbreviations: EDMD1 (EMD), patients with EMD gene mutation; EDMD2 (LMNA), patients with LMNA gene mutation; ESHF, end-stage heart failure; HF hosp, hospitalization for heart failure; HTX, heart transplant; RCA, resuscitated cardiac arrest

\section{A}

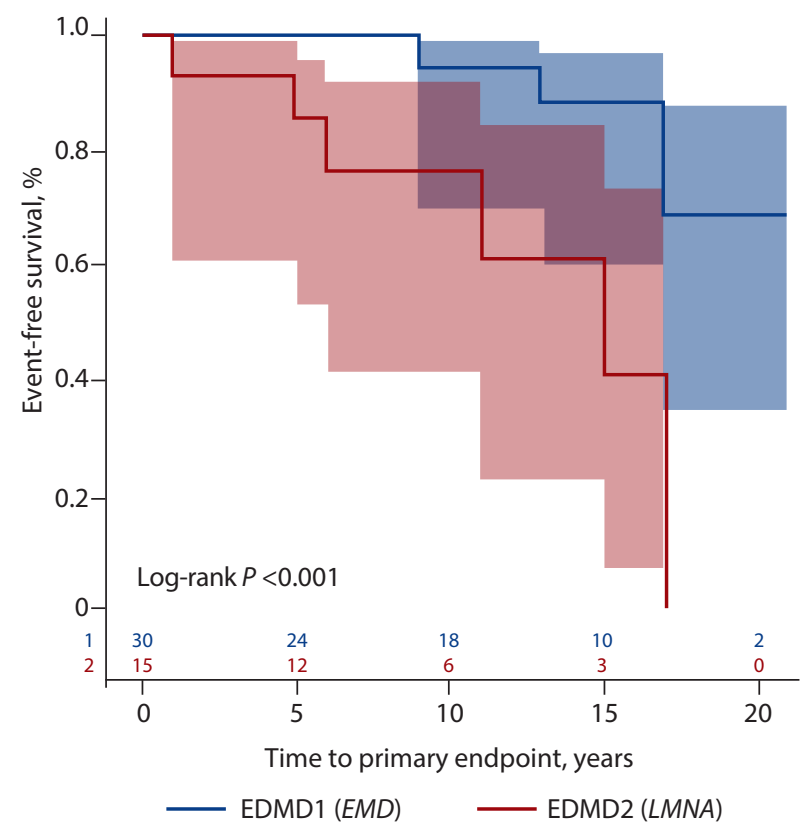

B

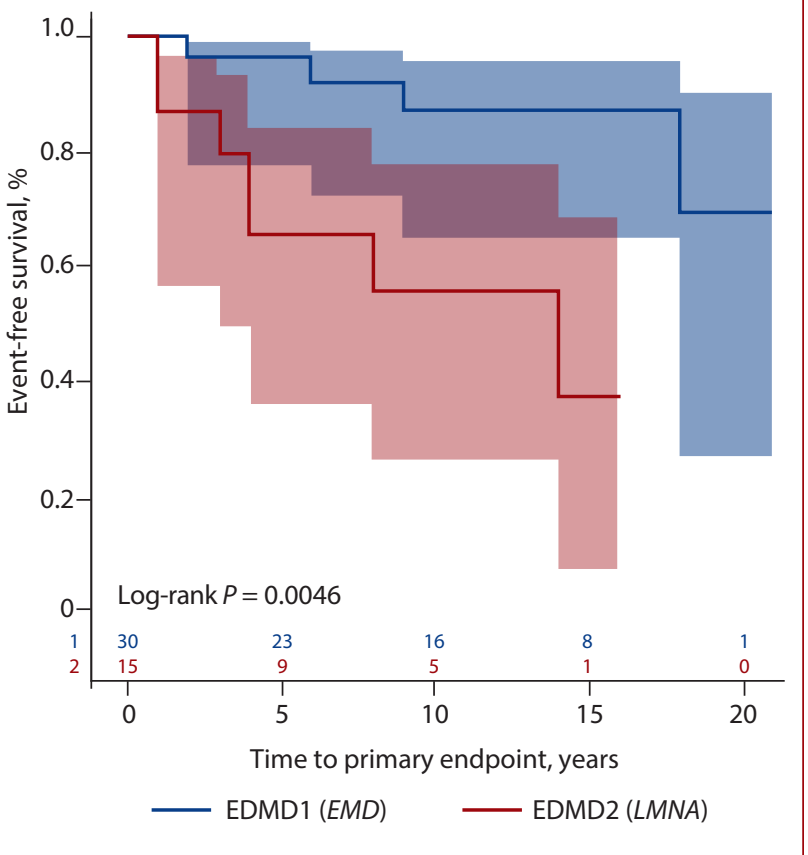

Figure 2. Kaplan-Meier survival curves for primary (A) and secondary (B) endpoint for both EDMD1 and EDMD2 Abbreviations: see Figure 1

posite secondary endpoint had higher atrial volumes (both left and right atrium) and more frequently were laminopathic patients. The presentation of HF symptoms (New York Heart Association [NYHA] class II-IV) at baseline also carried a higher risk of a clinical event occurrence (Table 2).

When stroke was analyzed as a separate endpoint, a trend in older patients suffering from stroke (40 [27-41] vs. 25 [16-34] years; $P=0.08$ ) was observed. Those patients more frequently had atrial standstill (AS) (50\% vs. 5\%; $P=0.02)$ and atrio-ventricular (AV) block ( $83 \%$ vs. $15 \%$; $P=0.002)$ at baseline. They also initially presented higher left atrial (LAV) and right atrial (RAV) volumes (LAV: 71 [67-117] vs. 53 [44-64] $\mathrm{ml} ; P=0.01$ and RAV: 114 [82-189] vs. 58 [44-88] $\mathrm{ml} ; P=0.02$ ).

In univariate analysis, several parameters were associated with a higher risk of death, including female gender, LMNA mutation, NT-proBNP, both systolic and diastolic 
Table 3. Predictors for primary and secondary composite endpoints

\begin{tabular}{|c|c|c|c|c|}
\hline \multirow[t]{2}{*}{ Variable } & \multicolumn{2}{|c|}{ Primary endpoint } & \multicolumn{2}{|c|}{ Secondary endpoint } \\
\hline & $\operatorname{HR}(95 \% \mathrm{CI})$ & P-value & HR $(95 \% \mathrm{Cl})$ & P-value \\
\hline Age, years & $1.00(0.96-1.06)$ & 0.90 & $1.02(0.98-1.06)$ & 0.50 \\
\hline Female sex & $6.61(1.27-34.4)$ & 0.02 & $1.68(0.61-4.65)$ & 0.30 \\
\hline LMNA mutation & $6.01(1.61-22.4)$ & 0.008 & $3.74(1.33-10.6)$ & 0.01 \\
\hline Non-missense mutation & $0.00(0.00-0.00)$ & 1.00 & $7.01(1.48-33.3)$ & 0.01 \\
\hline NYHA (II-IV) & $2.76(0.33-23.1)$ & 0.30 & $3.78(1.06-13.5)$ & 0.04 \\
\hline Sinus rhythm & $0.67(0.17-2.61)$ & 0.60 & $0.45(0.14-1.42)$ & 0.20 \\
\hline Atrial fibrillation & $0.70(0.15-3.30)$ & 0.60 & $0.88(0.28-2.76)$ & 0.80 \\
\hline AV block & $1.88(0.40-8.85)$ & 0.40 & $2.50(0.70-8.89)$ & 0.20 \\
\hline NT-proBNP, pg/ml & $1.00(1.00-1.00)$ & 0.01 & $1.00(1.00-1.00)$ & 0.046 \\
\hline NT-proANP, pmol/l & $0.91(0.49-1.71)$ & 0.80 & $1.04(0.62-1.73)$ & 0.90 \\
\hline LVEDdi, $\mathrm{mm} / \mathrm{m}^{2}$ & $1.31(1.03-1.68)$ & 0.03 & $1.29(1.07-1.56)$ & 0.008 \\
\hline LVESdi, $\mathrm{mm} / \mathrm{m}^{2}$ & $1.17(1.00-1.37)$ & 0.048 & $1.28(1.09-1.49)$ & 0.002 \\
\hline $\mathrm{LADi}, \mathrm{mm} / \mathrm{m}^{2}$ & $1.00(0.82-1.21)$ & 1.00 & $1.06(0.92-1.24)$ & 0.40 \\
\hline $\mathrm{LAVi}, \mathrm{ml} / \mathrm{m}^{2}$ & $0.99(0.95-1.03)$ & 0.50 & $1.01(0.98-1.03)$ & 0.50 \\
\hline LVEF, \% & $0.98(0.92-1.05)$ & 0.60 & $0.957(0.90-1.02)$ & 0.10 \\
\hline LVSF, \% & $0.95(0.89-1.02)$ & 0.20 & $0.927(0.87-0.99)$ & 0.02 \\
\hline $\mathrm{RVi}, \mathrm{mm} / \mathrm{m}^{2}$ & $1.04(0.85-1.27)$ & 0.70 & $1.06(0.91-1.23)$ & 0.60 \\
\hline $\mathrm{RAVi}, \mathrm{ml} / \mathrm{m}^{2}$ & $1.00(0.98-1.02)$ & 0.70 & $1.01(1.00-1.03)$ & 0.10 \\
\hline TAPSE, mm & $0.84(0.71-0.99)$ & 0.04 & $0.78(0.67-0.90)$ & $<0.001$ \\
\hline TVPG, mm Hg & $1.03(0.98-1.07)$ & 0.20 & $1.04(1.01-1.07)$ & 0.008 \\
\hline
\end{tabular}

Abbreviations: see Table 1

indexed left ventricular diameters, and right ventricular function (TAPSE). Non-missense mutation, HF symptoms (NYHA class II-IV), and left ventricular systolic function (LVSF) were additionally identified as predictors of the secondary endpoint (Table 3).

The presence of AS at baseline significantly increased the risk of stroke (hazard ratio [HR], 13.4; 95\% confidence interval [Cl], 2.24-80.7; $P=0.005$ ).

In a stepwise multivariable Cox regression analysis, LMNA mutation ( $\mathrm{HR}, 6.01 ; 95 \% \mathrm{Cl}, 1.61-22.4 ; P=0.008)$ and higher serum NT-proBNP concentration (HR, 1.29; $95 \%$ $\mathrm{Cl}, 1.06-1.56$ per $100 \mathrm{pg} / \mathrm{ml} ; P=0.01$ ) increased the risk of death. Higher TAPSE decreased the risk for the secondary endpoint (HR, 0.78; 95\% Cl, 0.68-0.90 mm; $P<0.001$ ).

NT-proBNP > $257 \mathrm{pg} / \mathrm{ml}$ (Area Under Curve [AUC], 0.75; sensitivity, 60\%; specificity, 88\%) and TAPSE <21 mm (AUC, 0.89 ; sensitivity, $85 \%$; specificity, $82 \%$ ) were identified as the best cut-off values for identifying the risk of death and secondary endpoint occurrence, respectively (Figure 3).

Kaplan-Meier curves for the secondary endpoint for EDMD patients depending on NT-proBNP serum concentration and TAPSE measurements are presented in Figure 4.

\section{DISCUSSION}

When first described, EDMD was considered as a benign form of muscular dystrophy $[5,6]$ as the progression of skeletal muscle involvement, especially in the case of EDMD1, is usually slow. Various degrees of cardiac involvement have been reported in the literature $[7,8]$. When the two main genetic types of EDMD — due to EMD and LMNA mutations - were identified [9-11], the differences in cardiological manifestation became clearer [12]. The risk was not only of bradyarrhythmias and AV blocks [13], which may be treated with pacemaker implantation [14] but also of atrial [13] and ventricular tachyarrhythmias with a typically very high risk of sudden death [15], dilated cardiomyopathy [16], HF [17] and stroke [18]. Typically, EDMD1 was associated with supraventricular arrhythmias and AV conduction abnormalities, while in EDMD2 cardiomyopathies, the risk of end-stage HF and sudden cardiac death was considered more common.

In our cohort, $36 \%$ of patients experienced clinical events during the median length of the ten-year (5-15) follow-up observation. Twenty-two percent of patients died during the follow-up. Taking into account the initial young age of the studied patients and other comorbidities, the overall risk should be considered very high. The risk of stroke in this population was also very high since $13 \%$ of the study participants developed this complication. Half of the patients who had a stroke (3/6) presented AS from baseline, and 4/6 were diagnosed with EDMD1 in the absence of classic stroke risk factors. AS, where no electrical and mechanical function of atria is present, was intuitively related to the risk of stroke. Our study adds more data to support this intuition in the EDMD population. Routinely used scales for the assessment of thromboembolic risk are probably not sufficient, and decisions regarding anticoagulation therapy in EDMD should be made individually, taking into account the presence of AS in particular.

It is worth pointing out that both EMD and LMNA patients developed events, although patients with EDMD2 were at a higher risk (HR, 3.74; 95\% Cl, 1.33-10.6; $P=0.01)$. In EDMD1 patients, the mortality rate was $13 \%$, while in the EDMD2 group $40 \%(P=0.02)$. Similarly, the 

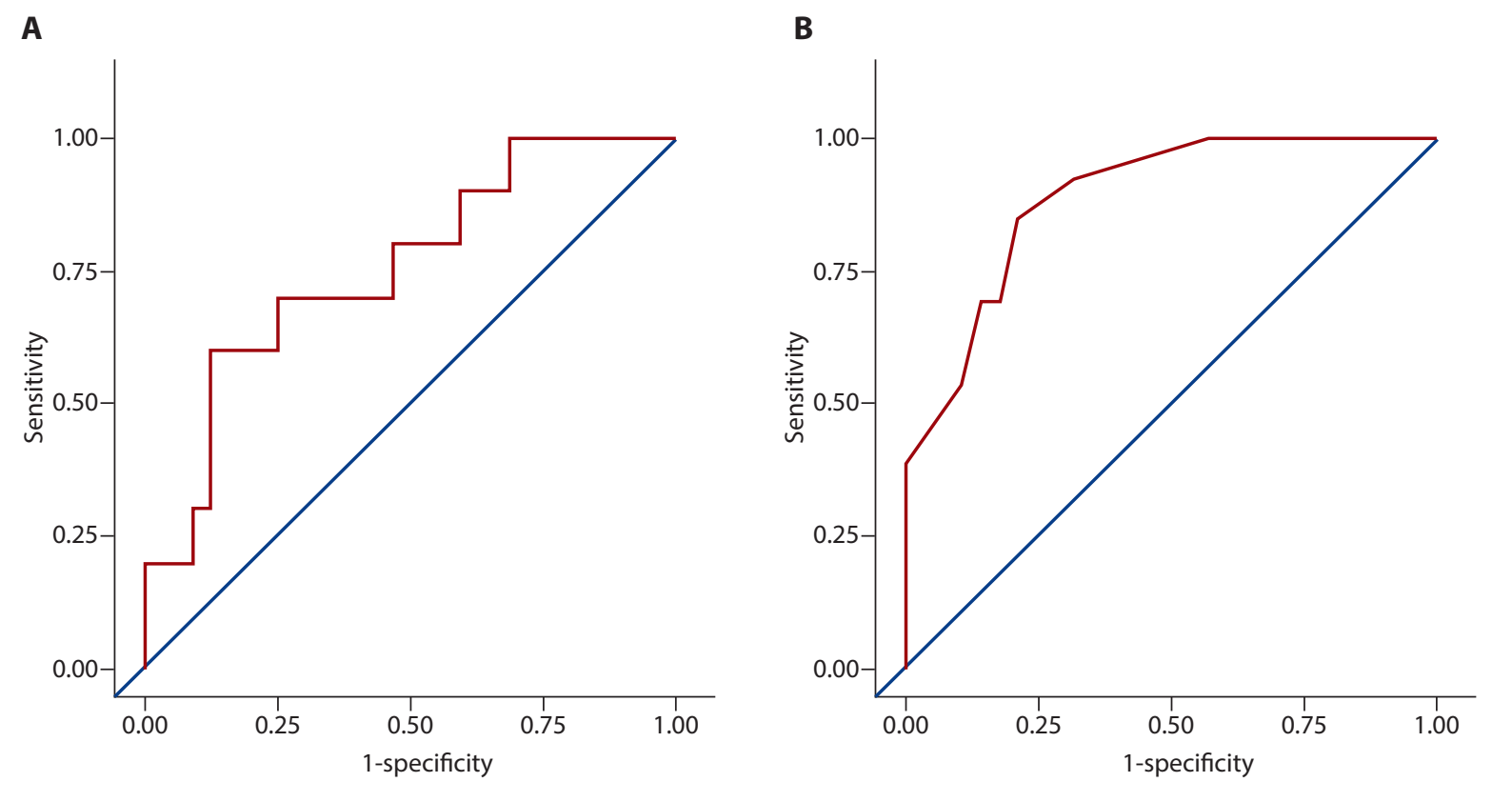

Figure 3. ROC curves for NT-proBNP for the primary endpoint (A) and TAPSE for the secondary endpoint (B)

Abbreviations: NT-proBNP, N-terminal fragment of B-type natriuretic peptide; ROC, receiver operating characteristic; TAPSE, tricuspid annular plane systolic excursion

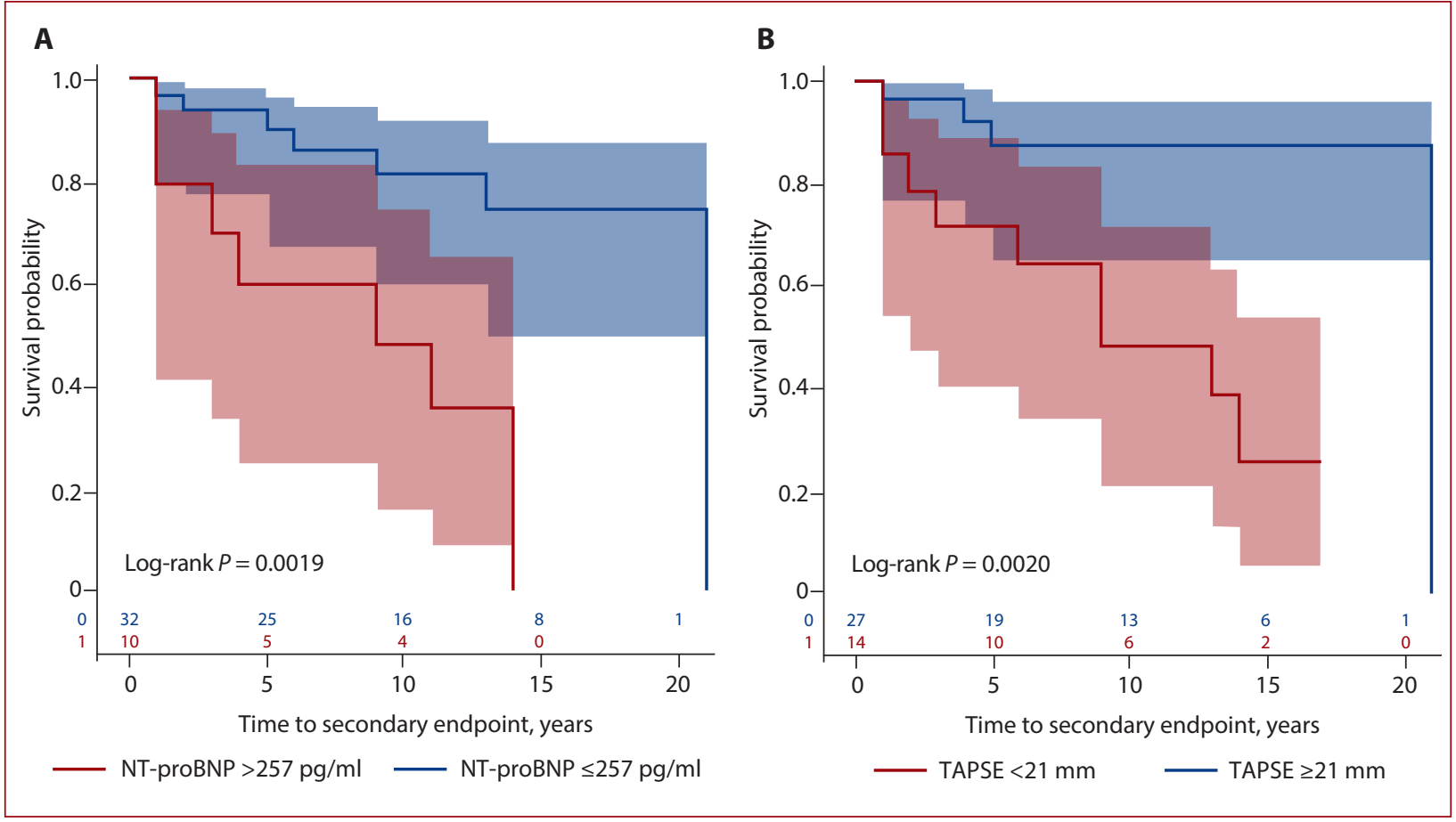

Figure 4. Kaplan-Meier survival curves for the secondary endpoint for EDMD patients depending on NT-proBNP serum concentration (A) and TAPSE measurements (B)

Abbreviations: see Figure 3 
risk for HF hospitalization was lower in the EDMD1 group than in the group EDMD2 (10\% vs. 33\%; $P=0.03)$. Only the incidence of stroke was comparable between EDMD1 and EDMD2.

LMNA mutation is a known risk factor for a malignant course and poor outcomes in dilated cardiomyopathy (DCM) [19]. However, in the setting of EDMD, this is a novel observation. High incidence of ventricular arrhythmias, the risk of sudden death [20,21], ESHF, and a need for HTX [22] are typical features of laminopathies with and without skeletal muscle involvement [23]. These observations were confirmed in our cohort. The majority of patients (six out of seven) who developed ESHF had mutations in the LMNA gene, including the two patients who received a heart transplant. Only one patient (3\%) in the EDMD1 group developed ESHF, and no HTX was needed in this group.

In a stepwise multivariable Cox regression analysis, higher serum NT-proBNP concentration increased the risk of patient death. The NT-proBNP is a well-known biomarker of cardiac dysfunction and HF. It is recommended in HF guidelines as a tool to diagnose and guide HF management. NT-proBNP $\geq 125 \mathrm{pg} / \mathrm{ml}$ is listed in the recently published universal definition and classification of HF [24]. The new study demonstrated the prognostic value of the natriuretic peptide in patients with a wide range of muscular dystrophies, although EDMD was not represented [25]. Natriuretic peptide levels were previously assessed in EDMD [26, 27], yet no prognostic information has been proven so far for this particular form of muscular dystrophy. Our study is the first in which the prognostic significance of NT-proBNP in this population has been proven. The NT-proBNP concentration of $257 \mathrm{pg} / \mathrm{ml}$ (AUC, 0.75) was determined as the best cut-off point for identifying the risk of death in EDMD. Although this level of NT-proBNP is not considered very high or might even be perceived as fitting in the upper normal limit for older patients or those with arrhythmias like AF, in such young patients with muscular dystrophy (median age, 25 [16-28] years) we should not overlook even slightly increased NT-proBNP since it may have a prognostic value. In our cohort, there was no significant difference in NT-proBNP between AF and no AF patients (94 [62-265] vs. 71 [45-167] pg/ml; $P=0.30$ ). Thus, AF (usually low symptomatic and with a low ventricular rate) is probably not responsible for increased NT-proBNP in some EDMD patients. Additionally, in our cohort for each $100 \mathrm{pg} / \mathrm{ml}$ of NT-proBNP increase, there was a $29 \%$ increase in the risk of death. This may be important in clinical practice while early identification of EDMD patients at risk of cardiovascular complication may lead to early detailed cardiovascular screening commonly carried out prior to symptomatic HF occurrence. Similarly, the results of the study by Mączyńska-Mazuruk et al. [28] conducted among patients with hypertrophic cardiomyopathy (603 patients; mean age, 44 years) have shown that patients with low NT-proBNP levels have a good prognosis, and the risk of death, heart transplantation, and hospitalizations due to heart failure significantly increase in patients with higher but still relatively low NT-proBNP level $>300 \mathrm{pg} / \mathrm{ml}$. Therefore, NT-proBNP may have important predictive value in genetic cardiomyopathies and should be considered in routine evaluation even in the young population.

Depressed left ventricular systolic function is a typical feature of cardiomyopathy in the course of dystrophinopathy [29] and laminopathy [22]. There is also evidence of both ventricles' involvement in cardiomyopathy when EDMD is diagnosed [3]. Interestingly, in our cohort, LVSF (left ventricular ejection fraction [LVEF]) as a left ventricular function parameter differed between those patients developing events and those who did not present events. Decreased LVSF was the only left ventricular function marker to be documented as a risk factor for the composite endpoint in univariate analysis. In stepwise multivariable Cox regression analysis, only higher TAPSE decreased the risk of reaching the secondary endpoint $(\mathrm{HR}, 0.78 ; 95 \% \mathrm{Cl}, 0.68-0.90 \mathrm{~mm}$; $P<0.001)$. The no other left ventricular function parameter carried the same significance. Preserved right ventricular function is crucial for adequate lung perfusion and blood collection from systemic circulation. TAPSE corresponds to the longitudinal distance of systolic excursion of the lateral tricuspid annulus toward the apex in a standard apical 4-chamber echocardiographic view. This was first described and validated by Kaul et al. [30] and is recommended as a routine and simple method of estimating RV function [31]. There is evidence of the prognostic value of TAPSE in a broad group of patients with cardiovascular disease; however, its significance was not tested in the EDMD population. The cut-off value for TAPSE as a predictor of outcomes was identified as $<21 \mathrm{~mm}$ (AUC, 0.89). This is much more than the recommended cut-off point for significant right ventricular dysfunction, which is $<17 \mathrm{~mm}$ [31]. This may mean that even mildly diminished right ventricular function, which we consider to be still within normal limits, represents an increased cardiovascular risk and indicates the need for clinical vigilance. Our data, therefore, suggest that right ventricular function estimation may be of great importance for future event prediction and that it outperforms traditional measures of left ventricular function in this respect.

\section{Study limitations}

The group of patients is relatively small, which is an evident limitation of the study, yet, given the extreme rarity of the disease, such a homogeneous group is still one of the largest ever reported. The total number of events is similarly small, which should be taken into account when interpreting the obtained results and their statistical significance.

\section{CONCLUSIONS}

In the cohort of EDMD patients, the presence of $L M N A$ mutation and higher NT-proBNP concentration were associated with increased mortality in the long-term follow-up. LowerTAPSE was an additional predictor of future adverse events in EDMD. 


\section{Article information}

Funding: The work was financed from funds granted as part of the statutory activity of the Medical University of Warsaw (1WR/N/2004).

\section{Conflict of interest: None declared.}

Open access: This article is available in open access under Creative Common Attribution-Non-Commercial-No Derivatives 4.0 International (CC BY-NC-ND 4.0) license, allowing to download articles and share them with others as long as they credit the authors and the publisher, but without permission to change them in any way or use them commercially. For commercial use, please contact the journal office at kardiologiapolska@ptkardio.pl.

How to cite: Marchel M, Madej-Pilarczyk A, Steckiewicz R, et al. Predictors of mortality and cardiovascular outcomes in Emery-Dreifuss muscular dystrophy in a long-term follow-up. Kardiol Pol. 2021;79(12): 1335-1342, doi: 10.33963/KP.a2021.0159.

\section{REFERENCES}

1. Heller SA, Shih R, Kalra R, et al. Emery-Dreifuss muscular dystrophy. Muscle Nerve. 2020; 61(4): 436-448, doi: 10.1002/mus.26782, indexed in Pubmed: 31840275.

2. Wang S, Peng D. Cardiac Involvement in Emery-Dreifuss Muscular Dystrophy and Related Management Strategies. Int Heart J. 2019; 60(1): 12-18, doi: 10.1536/ihj.17-604, indexed in Pubmed: 30518714.

3. Marchel M, Madej-Pilarczyk A, Tymińska A, et al. Echocardiographic features of cardiomyopathy in emery-dreifuss muscular dystrophy. Cardiol Res Pract. 2021; 2021: 8812044, doi: 10.1155/2021/8812044, indexed in Pubmed: 33614169.

4. Marchel M, Madej-Pilarczyk A, Tymińska A, et al. Cardiac arrhythmias in muscular dystrophies associated with emerinopathy and laminopathy: a cohort study.J Clin Med. 2021; 10(4), doi: 10.3390/jcm10040732, indexed in Pubmed: 33673224.

5. Emery AE, Dreifuss FE. Unusual type of benign $x$-linked muscular dystrophy. J Neurol Neurosurg Psychiatry. 1966; 29(4): 338-342, doi: 10.1136/jnnp.29.4.338, indexed in Pubmed: 5969090.

6. Rowland LP, Fetell $M$, Olarte $M$, et al. Emery-Dreifuss muscular dystrophy. Ann Neurol. 1979; 5(2): 111-117, doi: 10.1002/ana.410050203, indexed in Pubmed: 426473.

7. Bialer MG, McDaniel NL, Kelly TE. Progression of cardiac disease in Emery-Dreifuss muscular dystrophy. Clin Cardiol. 1991; 14(5): 411-416, doi: 10.1002/clc.4960140509, indexed in Pubmed: 2049891.

8. Buckley AE, Dean J, Mahy IR. Cardiac involvement in Emery Dreifuss muscular dystrophy: a case series. Heart. 1999; 82(1): 105-108, doi: 10.1136/hrt.82.1.105, indexed in Pubmed: 10377322.

9. Bione $S$, Maestrini E, Rivella S, et al. Identification of a novel X-linked gene responsible for Emery-Dreifuss muscular dystrophy. Nat Genet. 1994; 8(4): 323-327, doi: 10.1038/ng1294-323, indexed in Pubmed: 7894480.

10. Bonne G, Di Barletta MR, Varnous S, et al. Mutations in the gene encoding lamin A/C cause autosomal dominant Emery-Dreifuss muscular dystrophy. Nat Genet. 1999; 21(3): 285-288, doi: 10.1038/6799, indexed in Pubmed: 10080180.

11. Bonne G, Mercuri E, Muchir A, et al. Clinical and molecular genetic spectrum of autosomal dominant Emery-Dreifuss muscular dystrophy due to mutations of the lamin A/C gene. Ann Neurol. 2000; 48(2): 170-180, indexed in Pubmed: 10939567.

12. Madej-PilarczykA, Madej-PilarczykA, Kochański A. Emery-Dreifuss muscular dystrophy: the most recognizable laminopathy. Folia Neuropathol. 2016; 54(1): 1-8, doi: 10.5114/fn.2016.58910, indexed in Pubmed: 27179216.

13. Sakata K, Shimizu M, Ino H, et al. High incidence of sudden cardiac death with conduction disturbances and atrial cardiomyopathy caused by a nonsense mutation in the STA gene. Circulation. 2005; 111(25): 3352-3358, doi: 10.1161/CIRCULATIONAHA.104.527184, indexed in Pubmed: 15967842.

14. Steckiewicz R, Stolarz P, ŚwiętońE, et al. Cardiac pacing in 21 patients with Emery-Dreifuss muscular dystrophy: a single-centre study with a 39-year follow-up. Kardiol Pol. 2016; 74(6):576-583, doi: 10.5603/KP.a2015.0218, indexed in Pubmed: 26575312.

15. van Rijsingen IAW, Arbustini E, Elliott PM, et al. Risk factors for malignant ventricular arrhythmias in lamin a/c mutation carriers a European cohort study. J Am Coll Cardiol. 2012; 59(5): 493-500, doi: 10.1016/j. jacc.2011.08.078, indexed in Pubmed: 22281253.

16. Smith GC, Kinali M, Prasad SK, et al. Primary myocardial dysfunction in autosomal dominant EDMD. A tissue doppler and cardiovascular magnetic resonance study. J Cardiovasc Magn Reson. 2006; 8(5): 723-730, doi: 10.1080/10976640600723862, indexed in Pubmed: 16891232.

17. Madej-Pilarczyk A, Marchel M, Ochman K, et al. Low-symptomatic skeletal muscle disease in patients with a cardiac disease- Diagnostic approach in skeletal muscle laminopathies. Neurol Neurochir Pol. 2018;52(2): 174-180, doi: 10.1016/j.pjnns.2017.09.006, indexed in Pubmed: 28987496.

18. Boriani G, Gallina M, Merlini L, et al. Clinical relevance of atrial fibrillation/flutter, stroke, pacemaker implant, and heart failure in Emery-Dreifuss muscular dystrophy: a long-term longitudinal study. Stroke. 2003; 34(4): 901-908, doi: 10.1161/01.STR.0000064322.47667.49, indexed in Pubmed: 12649505

19. Tesson $F$, Saj $M$, Uvaize $M M$, et al. Lamin $A / C$ mutations in dilated cardiomyopathy. Cardiol J. 2014; 21(4):331-342, doi: 10.5603/CJ.a2014.0037, indexed in Pubmed: 24846508.

20. van Berlo JH, de Voogt WG, van der Kooi AJ, et al. Meta-analysis of clinical characteristics of 299 carriers of LMNA gene mutations: do lamin A/C mutations portend a high risk of sudden death? J Mol Med (Berl). 2005; 83(1): 79-83, doi: 10.1007/s00109-004-0589-1, indexed in Pubmed: 15551023.

21. Wahbi K, Ben Yaou R, Gandjbakhch E, et al. Development and validation of a new risk prediction score for life-threatening ventricular tachyarrhythmias in laminopathies. Circulation. 2019; 140(4): 293-302, doi: 10.1161/CIRCULATIONAHA.118.039410, indexed in Pubmed: 31155932.

22. Hasselberg NE, Haland TF, Saberniak J, et al. Lamin A/C cardiomyopathy: young onset, high penetrance, and frequent need for heart transplantation. Eur Heart J. 2018; 39(10): 853-860, doi: 10.1093/eurheartj/ehx596, indexed in Pubmed: 29095976.

23. Ditaranto R, Boriani G, Biffi M, et al. Differences in cardiac phenotype and natural history of laminopathies with and without neuromuscular onset. Orphanet J Rare Dis. 2019; 14(1): 263, doi: 10.1186/s13023-019-1245-8, indexed in Pubmed: 31744510.

24. Bozkurt B, Coats AJS, Tsutsui $\mathrm{H}$, et al. Universal definition and classification of heart failure: a report of the Heart Failure Society of America, Heart Failure Association of the European Society of Cardiology, Japanese Heart Failure Society and Writing Committee of the Universal Definition of Heart Failure: Endorsed by the Canadian Heart Failure Society, Heart Failure Association of India, Cardiac Society of Australia and New Zealand, and Chinese Heart Failure Association. Eur J Heart Fail. 2021; 23(3): 352-380, doi: 10.1002/ejhf.2115, indexed in Pubmed: 33605000.

25. Nikhanj A, Miskew Nichols B, Wang K, et al. Evaluating the diagnostic and prognostic value of biomarkers for heart disease and major adverse cardiac events in patients with muscular dystrophy. Eur Heart J Qual Care Clin Outcomes. 2021; 7(6):564-573, doi: 10.1093/ehjqcco/qcaa059, indexed in Pubmed: 32687175.

26. Niebroj-Dobosz I, Sokołowska B, Madej-Pilarczyk A, et al. Cardiovascular risk markers in dilated cardiomyopathy in Emery-Dreifuss muscular dystrophy (EDMD). Int J Cardiol. 2014; 173(2): 324-325, doi: 10.1016/j. ijcard.2014.03.058, indexed in Pubmed: 24681014.

27. Dobosz I, Sokołowska B. Natriuretic peptides assessment in dilated cardiomyopathy in patients with Emery-Dreifuss muscular dystrophy. Journal of Clinical \& Experimental Cardiology. 2012; 03(08), doi: 10.4172/21559880.1000206 .

28. Birnkrant DJ, Bushby K, Bann CM, et al. Diagnosis and management of Duchenne muscular dystrophy, part 2: respiratory, cardiac, bone health, and orthopaedic management. Lancet Neurol. 2018; 17(4):347-361, doi: 10.1016/S1474-4422(18)30025-5, indexed in Pubmed: 29395990.

29. Mączyńska-Mazuruk R, Małek ŁA, Kłopotowski M, et al. Serum Nterminal pro-brain natriuretic peptide as a prognostic marker in patients with hypertrophic cardiomyopathy. Kardiol Pol. 2019; 77(5): 571-573, doi: 10.5603/KP.a2019.0042, indexed in Pubmed: 30835329.

30. Kaul S, Tei C, Hopkins JM, et al. Assessment of right ventricular function using two-dimensional echocardiography. Am Heart J. 1984; 107(3): 526-531, doi: 10.1016/0002-8703(84)90095-4, indexed in Pubmed: 6695697.

31. Rudski L, Lai W, Afilalo J, et al. Guidelines for the echocardiographic assessment of the right heart in adults: a report from the American Society of Echocardiography. Journal of the American Society of Echocardiography. 2010; 23(7): 685-713, doi: 10.1016/j.echo.2010.05.010. 